\title{
Belphégor
}

\section{Relire Charles Grivel. Quelques réflexions en marge de «Alexandre Dumas : mal écrire, bien écrire »}

\section{Sarah Mombert}

\section{(2) OpenEdition}

1 Journals

\section{Electronic version}

URL: https://journals.openedition.org/belphegor/1290

DOI: $10.4000 /$ belphegor. 1290

ISSN: 1499-7185

Publisher

LPCM

\section{Electronic reference}

Sarah Mombert, "Relire Charles Grivel. Quelques réflexions en marge de "Alexandre Dumas : mal écrire, bien écrire »", Belphégor [Online], 16-1 | 2018, Online since 06 July 2018, connection on 19 October 2021. URL: http://journals.openedition.org/belphegor/1290 ; DOI: https://doi.org/10.4000/ belphegor.1290

This text was automatically generated on 19 October 2021 .

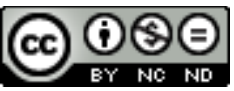

Belphégor est mis à disposition selon les termes de la Licence Creative Commons Attribution - Pas d'Utilisation Commerciale - Pas de Modification 4.0 International. 


\title{
Relire Charles Grivel. Quelques réflexions en marge de « Alexandre Dumas : mal écrire, bien écrire »
}

\author{
Sarah Mombert
}

\section{REFERENCES}

Charles Grivel, "Alexandre Dumas : mal écrire, bien écrire », dans Cent cinquante Ans après, Société des Amis d'Alexandre Dumas, 1995, p. 189-197.

Beaucoup de chercheurs en littérature du XIXe siècle partagent sans doute avec moi le souvenir de leur premier contact avec les écrits de Charles Grivel. Étudiante à peine sortie de classe préparatoire, je découvrais à l'université que le monde des études littéraires ne cessait pas de tourner si l'on abandonnait la sainte trinité lagarde-etmichardienne de l'homme, l'œuvre et la chronologie. Si les étudiants de l'ère poststructuraliste se passionnaient pour la théorie littéraire, empruntaient sans vergogne la boîte à outils des sciences humaines, osaient proposer des sujets de mémoires nonmonographiques, ils le devaient à la lecture de textes tels que Production de l'intérêt romanesque ${ }^{1}$, le livre tiré de la thèse de Charles Grivel. La relecture récente des notes prises jadis sur ce livre touffu et stimulant, d'une ambition théorique remarquable, montre que je n'avais pas compris grand-chose au détail de la démonstration. Mais ce que j'ai immédiatement perçu, et qui m'a beaucoup inspirée, c'est la façon dont Charles Grivel y dessinait un corpus immense (deux cents romans), choisi dans une tranche synchronique (la décennie 1870-1880), auquel il posait des questions fondamentales : pourquoi raconte-on? pourquoi aime-t-on lire des histoires? sur quoi repose l'attrait de la fiction?

2 En 1994, lors d'un colloque organisé à Marly-le-Roi par la Société des Amis d'Alexandre Dumas, j'ai entendu Charles Grivel prolonger les réflexions théoriques de son livre de 1973 en s'interrogeant sur le «cas» Dumas. Le texte de cette communication, publié 
sous le titre "Alexandre Dumas: mal écrire, bien écrire", constitue le noyau du premier chapitre du livre Alexandre Dumas, l'homme 100 têtes $^{2}$, qui rassemble trois décennies de travaux consacrés à cet auteur. Il ouvre la quête du « secret » dans lequel s'origine, selon Charles Grivel, l'écriture dumasienne, et que ne cessent de dire et de cacher à la fois les œuvres de l'écrivain, comme le motif dans le tapis de la nouvelle de Henry James. Je n'ai jamais été absolument convaincue que l'on doive, comme le fait Charles Grivel, attribuer à la question raciale la première place dans ce « secret ». Mais cela ne m'empêche pas d'apprécier et d'admirer la démarche qui consiste à tenter de comprendre les mécanismes profonds de la "compulsion d'écriture», en les reliant intimement au déni de reconnaissance qu'oppose obstinément à Dumas l'institution littéraire, contre l'avis des lecteurs. Quel que soit le « secret » que l'on identifie comme la source de cette pulsion d'écriture, il est certain en effet qu'elle a quelque chose à voir avec l'identité d'écrivain, conquise de haute lutte par l'autodidacte Dumas et incessamment réaffirmée par l'exercice d'un savoir-faire qui, à défaut de la légitimité académique, lui vaut la reconnaissance du public.

3 La réception de Dumas ne pouvait qu'intéresser Charles Grivel, qui fut l'assistant du théoricien de l'esthétique de la réception Hans-Robert Jauss. Ce n'est, dans « Mal écrire, bien écrire ", que le point de départ d'une exploration des mutations profondes de la littérature à l'ère démocratique, mais un point de départ indispensable. Charles Grivel formule clairement le paradoxe de la réception de Dumas mais aussi, au-delà de ce cas particulier, de toutes les œuvres produites dans le contexte des industries culturelles nées au XIX ${ }^{\mathrm{e}}$ siècle : comment et pourquoi se trouve-t-il systématiquement minoré par l'institution littéraire (la critique, l'Académie, l'université), alors même que ni sa notoriété ni l'intérêt des lecteurs pour son œuvre n'ont faibli depuis deux siècles?

Charles Grivel fonde son analyse sur un florilège de citations, dont la richesse et la variété témoignent de sa curiosité de lecteur. Dumas, remarque Grivel, «a fait face à une campagne de dénigrement d'une rare ampleur, de durée remarquable, de singulière nature ${ }^{3}$ ", qui débute dans les années 1840 et se prolonge jusqu'à aujourd'hui. Le nom, universellement connu, de Dumas et les personnages inoubliables qu'il a inventés, sont systématiquement accompagnés, sous la plume des écrivains et critiques dont Grivel recueille des extraits (Jean-Claude Pirotte, Didier Daencinckx, etc.), par un cortège de jugements de valeur et d'images associées qui introduit autant de restrictions dans l'appréciation de son héritage culturel et met en doute son écriture même. La lecture de Charles Grivel, attentive aux «implications, [aux] formules à moitié formulées, [aux] réticences ${ }^{4} »$ du discours critique, dévoile toutes les nuances du mépris social qui s'expriment dans le portrait de l'homme : racisme, lorsqu'on dénonce la vanité du descendant d'esclaves et le caractère « africain » de son génie littéraire, ou puritanisme envers l'homme à femmes, grand admirateur des beautés féminines et masculines.

5 L'évaluation critique de l'écriture de Dumas pâtit des mêmes préjugés informulés car, souligne Charles Grivel, « le déni frappe [...] à la fois l'homme et l'œuvre ${ }^{5}$ ». Au premier rang des promoteurs de la "littérature industrielle» dénoncée par Sainte-Beuve ${ }^{6}$, Dumas « est un faiseur professionnel et non pas un inspiré ${ }^{7}$ » et le mode de production littéraire qu'il adopte dans les années 1840 contribue à dégonfler le mythe romantique du génie. L'ampleur inouie de son œuvre, la foule de ses personnages, au centre de laquelle se campe solidement l'inventeur fier de sa propre fécondité, apparaissent aux yeux des critiques du XIX ${ }^{e}$ siècle comme une tentative pour monopoliser la production 
littéraire nationale, pour s'approprier le succès et rassembler autour de sa gigantesque personne tous les suffrages d'un public trop aisé à séduire. Premier écrivain d'une ère démocratique et mercantile, Dumas «se donne pour tâche d'écrire tous les livres, d'écrire à tous, toutes opinions confondues, tout ce qu'ils désirent lire. La littérature universelle répond en lui à l'universelle demande ${ }^{8}$ ».

6 Les implications politiques de cette ambition universelle du grand vulgarisateur qui, disant « je », prétend « écrire les mémoires de la France ${ }^{9}$ ", n'échappent pas à Charles Grivel. Le césarisme plébiscitaire du Second Empire, en particulier, ne peut s'accommoder de ce qu'incarne Dumas : « la volonté générale du Peuple mise en fiction et fondue en littérature ${ }^{10}$ ». L'analyse politico-économique de Grivel ne s'embarrasse pas des détails et ne prend guère en compte l'évolution de l'écrivain au cours de sa longue carrière. On pourrait lui opposer quelques nuances, par exemple l'idée que l'hostilité de l'empereur à Dumas provient autant, sinon plus, de son amitié incessamment proclamée pour Victor Hugo que de son rapport au peuple, ou que le mode de production littéraire de Dumas détonne moins dans un régime industrialiste que sa fidélité au romantisme social de sa jeunesse. Cela n'enlève rien à la justesse de la formule par laquelle Charles Grivel résume le dilemme imposé à l'écrivain par son époque: "D'un côté, elle fait du livre un produit, de l'autre, elle lui recommande d'échapper aux lois de la production ${ }^{11} »$.

7 La question de la collaboration littéraire est évidemment au cœur des attaques portées à Dumas, et Charles Grivel n'esquive nullement cet aspect, très polémique, de la réception de l'œuvre. Là encore, son propos n'est pas d'apporter de nouveaux éléments (à charge ou à décharge) au dossier, mais de souligner les implicites, en particulier moraux, du réquisitoire de l'accusation. Il remarque que, parmi les multiples exemples choisis par Genette dans Seuils ${ }^{12}$, Dumas illustre à lui seul la catégorie du "plagiat consenti». Il note encore que l'hommage paradoxal à Dumas rendu par le roman d'Arturo Pérez-Reverte Le Club Dumas ${ }^{13}$ repose tout entier sur l'argument d'un manuscrit qui prouverait que le «nègre " Auguste Maquet est le véritable auteur des Trois Mousquetaires.

8 Le recueil des innombrables témoignages de ce que Grivel appelle le « déni d'écriture " pourrait paraître quelque peu paranoïaque s'il ne marquait l'indispensable point de départ d'une réflexion très stimulante sur l'écriture de l'Histoire comme compilation. Récrire le passé à sa façon, s'approprier la représentation de la mémoire nationale pour en faire la matière d'une fiction constitue un premier attentat à la dignité de l'Histoire commis par Dumas. Soumettre la science patiente de l'archiviste et de l'historien aux modes de production de la littérature populaire, «vite faite, vite lue [...] tous les jours, en tous lieux et pour tous les goûts ${ }^{14} »$ en est un autre. Le troisième, et peut-être le plus grave, consiste à faire passer l'enseignement par l'amusement, à donner le passé national en pâture quotidienne aux abonnés du journal, au risque que la fiction le prive de sa grandeur patrimoniale : le récit n'a alors plus de «fonction cathartique, ce n'est plus un $m a n a^{15}$ ». Ancien étudiant en anthropologie culturelle, Charles Grivel analyse avec une grande pertinence le changement profond apporté par Dumas dans la littérature française : «Avec Dumas, le roman descend du ciel sur la terre, il opère sa mutation bourgeoise, il est devenu une façon de lire son journal et de vivre pareillement à tout ce qui s'y trouve écrit ${ }^{16}$. » L'avènement de « l'ère médiatique ${ }^{17}$ » qui, du XIX ${ }^{\mathrm{e}}$ siècle à aujourd'hui, bouleverse non seulement nos façons de lire, mais aussi nos représentations du monde et nos modes de communication, n'est pas sans lien avec 
ce qu'une société convient d'appeler « littérature ». Charles Grivel montre que l'œuvre de Dumas, en imposant de nouveaux critères d'appréciation de "l'écriture ", fait vaciller les quatre grands piliers du Temple culturel qu'il identifie comme «l'Histoire, la Littérature, la Personne et le $\mathrm{Moi}^{18}$ ».

Les études dumasiennes, qui s'implantent depuis deux décennies à l'université, doivent beaucoup aux travaux de Charles Grivel et à ses intuitions. Toujours attentif à la dimension communicationnelle du récit dumasien, Charles Grivel souligne volontiers ${ }^{19}$ la présence de l'oralité du conteur et les figurations dans le récit romanesque d'une narration adressée (narrateurs seconds, lettres, manuscrits trouvés). Il pointe ainsi l'une des sources majeures de l'émerveillement du lecteur par le merveilleux moderne que Julie Anselmini étudie dans Le Roman d'Alexandre Dumas père ou la Réinvention du merveilleux $^{20}$. Les réflexions de Charles Grivel sur l'incroyable fécondité de Dumas comme inventeur de mythes modernes trouvent leur prolongement dans le livre - qu'il aurait probablement aimé lire - de Maxime Prévost, Alexandre Dumas mythographe et mythologue ${ }^{21}$.

Plus généralement, sa façon de questionner la littérature sans emprunter les filtres usuels du jugement esthétique, pour éclairer les mécanismes profonds de la lecture de fiction, constitue une source de vive inspiration pour ceux qui, dans son sillage, ambitionnent de dépasser le canon étroit de ce qui s'enseigne. Parmi les questions qu'il a mises au jour et qui continuent à nourrir la recherche actuelle se trouve celle du volume du texte, de l'excès, du flux du récit, qu'il a identifiée depuis longtemps comme faisant partie de la séduction du genre romanesque : « Le romanesque est, entre autres, mais nécessairement, fonction de l'étendue du texte, l'excès de brièveté le stoppe ou le dilue $^{22}$. » L'analyse de la démesure de la littérature populaire, qui piège le lecteur par le renouvellement incessant du plaisir du texte, va de pair chez Charles Grivel avec une conception anti-essentialiste $\mathrm{du}$ genre romanesque. Le roman, affirme-t-il en substance, ne se définit pas par un ensemble d'invariants formels, de règles explicites ou implicites, de recettes qu'il suffirait d'appliquer, mais il est une série, constituée par les livres reçus comme romans par les lecteurs. Cette conception non-normative du genre romanesque - en régime populaire et bien au-delà - nourrit profondément les recherches actuelles sur la sérialité de la fiction, par exemple le récent livre de Matthieu Letourneux, Fictions à la chaîne ${ }^{23}$.

11 Charles Grivel abordait la modernité littéraire avec la passion d'un intellectuel et d'un collectionneur plus qu'avec la rigueur d'un historien. Bien que bon connaisseur des contextes historiques et culturels des œuvres qu'il étudiait, il les approchait rarement sous l'angle de l'histoire littéraire. Ce qui motivait toutes ses analyses, c'était une batterie de questions essentielles dont il avait posé la théorie dans son livre de 1973 et qu'il remettait sur le métier dans chaque étude de cas : pourquoi écrit-on? pourquoi lit-on? quels sont les mécanismes de l'attraction exercée par la fiction? Des questions que, trop souvent, les chercheurs s'abstiennent de poser, préférant prudemment s'en tenir aux objets d'érudition qui valorisent le savoir positif, camouflent leurs enjeux idéologiques et ne fâchent personne. Charles Grivel jouait volontiers de sa position légèrement décentrée d'universitaire franco-suisse professant dans une université allemande pour se permettre de poser aux chercheurs français des questions dérangeantes sur la littérature de leur pays.

En écoutant parler Charles Grivel, en le voyant tourner sans les lire ses petits feuillets empilés, recouverts d'une écriture illisible par quiconque d'autre que lui-même, on 
percevait immanquablement le charme et les difficultés d'une pensée qui s'approfondit en se partageant. Comme son écriture, sa parole n'était pas toujours limpide, mais elle était éclairante et suggestive. L'écouter naguère, le lire aujourd'hui, invite à explorer les innombrables et fécondes pistes qu'il ouvre. Il n'épuise jamais une intuition, ne pousse pas jusque dans les détails sa démonstration, mais sa pensée est un inépuisable gisement de suggestions pertinentes, de points de vue originaux, de propositions théoriques stimulantes, qui incitent chacun à réfléchir et à lire. Entre particulier à lire et à relire Dumas, sur les pas de Charles Grivel.

\section{NOTES}

1. Charles Grivel, Production de l'intérêt romanesque. Un état du texte (1870-1880), un essai de construction de sa théorie, La Hague-Paris, Mouton, « Approaches To Semiotics », 1973.

2. Charles Grivel, Alexandre Dumas, l'homme 100 têtes, Presses universitaires du Septentrion, « Objets », 2008.

3. Ibid., p. 190.

4. Ibid., p. 192.

5. Id.

6. Charles-Augustin Sainte-Beuve, «De la littérature industrielle », Revue des Deux-Mondes, t. 19, 1839, pp. 675-691.

7. Charles Grivel, art. cité, p. 192.

8. Ibid., p. 193.

9. Alexandre Dumas, Mes Mémoires, chap. CXXXVIII.

10. Charles Grivel, art. cité, p. 193.

11. Ibid.

12. Gérard Genette, Seuils, Seuil, « Poétique », 1987.

13. Arturo Pérez-Reverte, Le Club Dumas ou L'Ombre de Richelieu, trad. de l'espagnol par Jean-Pierre Quijano, J.-Cl. Lattès, 1994.

14. Charles Grivel, art. cité, p. 197.

15. Ibid.

16. Ibid.

17. Marie-Ève Thérenty et Alain Vaillant, 1836, l'an I de l'ère médiatique. Analyse littéraire et historique de La Presse d'Émile de Girardin, Nouveau Monde Éditions, 2001.

18. Charles Grivel, art. cité, p. 197.

19. Voir par exemple "Alexandre Dumas: le bas-narrer littéraire ", dans Richesses du roman populaire, éd. par René Guise et Hans-Jörg Neuschäfer, Centre de recherches sur le roman populaire de l'université de Nancy II, 1986, p. 293-314, et, pour un développement plus théorique, Production de l'intérêt romanesque, op. cit., p. 152 sqq.

20. Julie Anselmini, Le Roman d'Alexandre Dumas père ou la Réinvention du merveilleux, Genève, Droz, « Histoire des idées et critique littéraire », 2010.

21. Maxime Prévost, Alexandre Dumas mythographe et mythologue. L'Aventure extérieure, Honoré Champion, « Romantisme et Modernités », 2018.

22. Charles Grivel, Production de l'intérêt romanesque, op. cit., p. 36.

23. Matthieu Letourneux, Fictions à la chaîne : littératures sérielles et culture médiatique, Seuil, 2017. 
INDEX

Mots-clés: Grivel, Alexandre Dumas, littérature populaire

\section{AUTHOR}

SARAH MOMBERT

École Normale Supérieure de Lyon

UMR 5317 IHRIM

Université de Lyon 